\title{
Cartazes urbanos acessíveis a pessoas cegas: proposta de um modelo de design para todos, baseado numa solução mobile
}

\author{
Accessible urban posters to blind people: a proposal for a \\ design template for all, based on a mobile solution
}

Joana Pimparel Mineiro, Ana Margarida Almeida, Catarina Lélis

acessibilidade, cegos, cartazes, espaço urbano, design para todos, soluções mobile accessibility, blind, posters, urban space, design for all, mobile solutions

\begin{abstract}
O presente artigo apresenta uma investigação em curso cujo principal propósito é explorar a área da acessibilidade a pessoas cegas, procurando compreender como um cego consegue "ver" um cartaz de rua. Pretende-se conhecer e estudar como estes indivíduos interagem com o espaço urbano e compreender quais as melhores estratégias e paradigmas de interação a utilizar neste contexto. A principal finalidade é, pois, contribuir para a construção de um espaço urbano mais acessível, como forma de proporcionar a divulgação e a inclusão de todos. Assim, de forma a analisar e validar estas melhores estratégias, foi construído um protótipo, baseado numa solução mobile, em que a sua função é, sem dúvida, o ponto-chave e dominante neste processo. Nas diferentes secções do artigo apresentamos o enquadramento desta temática, o processo de desenvolvimento do referido protótipo assim como a metodologia utilizada durante o seu desenvolvimento e as conclusões perante a observação dos dados recolhidos no sistema adotado.
\end{abstract}

This article describes an on-going investigation that explores the field of accessibility to blind people, trying to understand how they can "see" a street poster. This investigation aims to study how these individuals interact with urban space and understand what are the best strategies and interaction paradigms to use in this context. The main purpose is to contribute to the construction of a more accessible urban space, in order to provide disclosure and inclusion for all. Therefore, in order to analyze and validate these best strategies, a prototype was built based on a mobile solution, where its function is undoubtedly the main point of the process. The different sections of this article present the theoretical background, the development process of the prototype as well as the methodology used and the conclusions before the observation of the data collected in the adopted system. 


\section{Introdução}

Hoje em dia as cidades são consideradas cenários em permanente comunicação com o ser humano, o qual com elas comunica através dos sentidos. A tentativa de os promover torna-se, por conseguinte, inquietante (Berns, 2002). O poder da imagem, do som, do cheiro, das texturas - através dos sinais de trânsito, semáforos, publicidade de rua, graffitis, toldos, letreiros de estabelecimentos, entre outros - são exemplos que dão voz, cor e sentido(s) à nossa vida. Contudo, devido ao excesso de informação, estas formas de comunicar tornam-se, frequentemente, banais, saturadas e, muitas vezes, difíceis de cativar.

Devido ao seu impacto, posição estratégica e à incorporação com o espaço urbano, a publicidade pode ter variadíssimos suportes (fixos ou móveis) e formatos, sendo considerada uma técnica de comunicação privilegiada devido à sua diversidade no espaço urbano (Moles, 2005). A vivência de cada um e a sua experiência sensível é, em parte, representada por estas imagens publicitárias em determinados contextos. Os cenários urbanos estão em constante alteração, não só promovida pela evolução da natureza publicitária, como pela forma como se relacionam com o meio, isto é, com os suportes físicos a que são as mensagens são aplicadas, permitindo assim uma dinâmica no espaço urbano.

Os pressupostos desta investigação baseiam-se na promoção da acessibilidade para pessoas cegas, nomeadamente no estudo e criação de soluções que favoreçam a compreensão, por parte do indivíduo cego, de um cartaz de rua. Pretende-se, portanto, compreender e analisar as interações do indivíduo cego com o espaço urbano, por forma a potenciar a identificação das melhores estratégias para a divulgação clara, para todos os indivíduos, de informação útil que seja efetivamente acessível e que permita a integração dos indivíduos num ambiente sem exclusão social.

"A pessoa portadora de deficiência deve receber uma educação que lhe permita adaptar-se ao ambiente que a rodeia e também encontrar o caminho e os meios que lhe permitem adaptar-se às situações futuras" (Canziani, 1985 p.47).

Estimativas de 2014 apontam para que, globalmente, existam cerca de 285 milhões de pessoas com algum tipo de deficiência visual, das quais 39 milhões sejam cegas e 246 milhões tenham baixa visão, números que têm tendência a aumentar em função dos problemas associados ao aumento da esperança média de vida (World Health Organization, 2014). Muitas destas pessoas utilizam software de acessibilidade específico (como lupas digitais e leitores de ecrã) para transformar a informação textual disponível nas interface gráficas dos equipamentos computacionais, num formato que possam interpretar com outros sentidos (por exemplo, a audição). Com efeito, numa 
sociedade globalizada, a palavra de ordem é a acessibilidade; no entanto, quando se publica um cartaz, este não é concebido tendo em conta indivíduos com algumas limitações, em particular com incapacidade visual.

Ora, numa sociedade inclusiva é importante a tomada de consciência de que qualquer informação, serviço ou sistema deve chegar a todo e qualquer cidadão, sendo fundamental estudar e implementar estratégias de Design para Todos, que promovam práticas de desenho para a inclusão social e para a igualdade (Declaração de Estocolmo, 2004). É através de lógicas de "utilização universal" (Shneiderman, 2002) das tecnologias e sistemas de informação que se poderá promover o acesso alargado destas soluções a qualquer indivíduo, diminuindo as dificuldades de interação com o meio envolvente e proporcionando o desenvolvimento de novos conhecimentos. Cabe ao designer compreender as necessidades do utilizador e responder a esses desafios criando produtos acessíveis, úteis e satisfatórios para um maior número de pessoas. Norman (2004) refere que um design baseado nas necessidades do utilizador coloca de parte questões estéticas e secundárias, simplificando tarefas, possibilitando assim uma abordagem positiva e controlada. É no âmbito particular dos cartazes urbanos, e tendo estes como principal função publicitar e divulgar informação de forma visual (Moles, 2005), que surge o projeto aqui descrito, PUB4ALL, o qual pretende promover a inclusão, através da criação de cartazes urbanos acessíveis a cegos pela apresentação de uma solução tecnológica que permita o acesso à informação de uma forma simples, prática e funcional.

\section{Metodologia}

A principal abordagem metodológica deste trabalho é a "investigação de desenvolvimento" (Van der Maren, 1996), na qual os processos de "especificação" e "prototipagem" assumiram especial relevância. Com efeito, foi definido como objetivo específico deste projeto a concretização de um protótipo demonstrativo de um cartaz de rua acessível a cegos, que possa ser validado por elementos representativos do público-alvo final e que permita a apresentação de um conjunto de princípios orientadores para a construção de cartazes de rua acessíveis (não apenas a cegos mas também ao público em geral).

No que respeita ao processo de especificação, e com o intuito de desenhar um modelo de sistema o mais aproximado possível das reais necessidades dos cegos, foi aplicado um inquérito por entrevista a um cego. Com base nos dados recolhidos com este instrumento foi possível especificar um modelo para o sistema a desenvolver e avançar para a etapa de prototipagem em que o protótipo desenvolvido seria implementado num espaço público acessível a todos. 
Durante o processo de validação do protótipo foi utilizado um guião de tarefas de apoio às sessões de experimentação, uma grelha de observação e um inquérito por questionário, inicial e final. Estas sessões foram alvo de registo em vídeo e áudio, apenas para efeito de validação e confirmação de dados.

\section{Especificação e prototipagem}

\subsection{0 cartaz}

Para efeitos de validação do protótipo desenvolvido, foi necessário produzir um cartaz (figura 1). Este representa um teaser do projeto, recorrendo assim a uma ferramenta usada em publicidade com o intuito de chamar a atenção para uma campanha publicitária. O teaser tem como função despertar a atenção e aumentar o interesse de um determinado público-alvo para uma mensagem, normalmente apresentada no início da campanha, através de informações enigmáticas (Viana, 2010), visando lançar uma ideia e criar uma certa expectativa, inquietação e curiosidade relativamente ao conteúdo da mensagem. No caso do cartaz desenvolvido, a interrogação do cidadão sobre a mensagem que pretende ser passada - como seria possível ver imagens sem as conseguir ver - emerge da imagem. A escolha de um olho e do slogan "Imagem que um olhar não lê" enformam o contexto esclarecido no rodapé do cartaz: Protótipo do projecto "Cartazes Urbanos Acessíveis a Cegos - Estratégias e Paradigmas de Interação” no âmbito do Mestrado de Comunicação Multimédia da Universidade de Aveiro. Concebido de forma a analisar e validar as melhores estratégias para a construção de um cartaz urbano acessível a todos.

Figura 1 Cartaz a utilizar na validação do protótipo

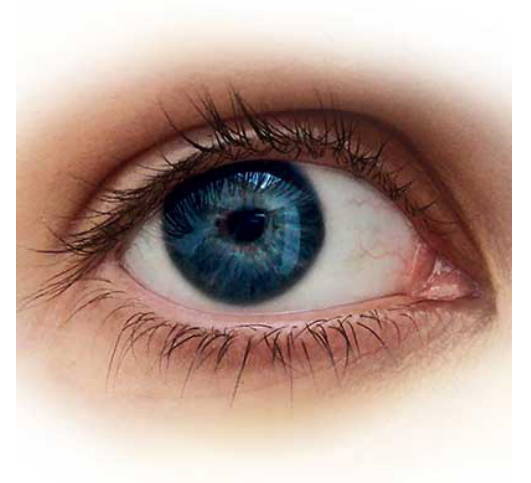

\section{IMAGENS}

que UM OLHAR NÃO LÊ

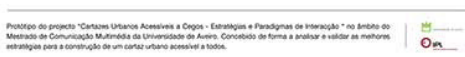


Mineiro, J. P., Almeida, A. M. \& Lélis, C. | Cartazes urbanos acessíveis a pessoas cegas: proposta de um modelo de design para todos, baseado numa solução mobile

\subsection{Espaço}

A cidade é o cenário adequado à aplicação deste protótipo. Todas as componentes que o formam e definem são o ponto-chave para validação dos resultados. Foi neste pressuposto que foi definido o cenário experimental que foi construído para o efeito: a parede exterior de um edifício público, nas instalações de um campus académico, no Instituto Politécnico de Leiria, em Portugal.

No que respeita ao percurso de exploração e experimentação do protótipo é necessário realizar um percurso de 60 metros, sendo que do ponto de partida até ao local de instalação do cartaz serão percorridos 40 metros (ação). Os restantes são apenas a continuação do caminho de saída do campus.

Figura 2 Descrição do percurso
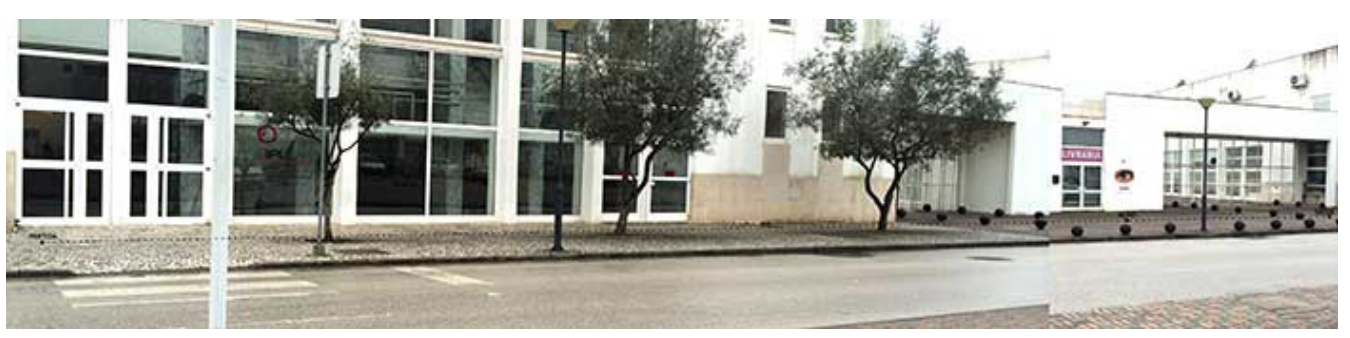

\subsection{Modelo de sistema e desenvolvimento do protótipo}

A construção do modelo de sistema foi realizada com base nos resultados obtidos com a aplicação de um inquérito por entrevista a um cego congénito. Com esta entrevista foi possível efetuar o levantamento dos mecanismos e fatores mais relevantes no processo de acesso à informação em contexto urbano nos indivíduos cegos.

Para o desenvolvimento deste protótipo foram testadas várias tecnologias e foi feito um levantamento de requisitos:

Tabela 1 Requisitos do protótipo

\begin{tabular}{|l|l|}
\hline Termo & Descrição \\
\hline Ponto de acesso & $\begin{array}{l}\text { Sistema que fornece o ponto de acesso ao } \\
\text { serviço }\end{array}$ \\
\hline Utilizador & $\begin{array}{l}\text { Utilizador, detentor de um telemóvel, que } \\
\text { interage com a solução }\end{array}$ \\
\hline Cliente/Dispositivo Receptor & $\begin{array}{l}\text { Sistema computacional do utilizador que } \\
\text { pretende aceder ao serviço }\end{array}$ \\
\hline Cartaz & Objeto que o utilizador pretende consultar \\
\hline
\end{tabular}


Mineiro, J. P., Almeida, A. M. \& Lélis, C. | Cartazes urbanos acessíveis a pessoas cegas: proposta de um modelo de design para todos, baseado numa solução mobile

Requisitos funcionais:

- O sistema deve apresentar o formato acessível de um cartaz físico;

- O sistema deve ser acessível a partir de um cliente do utilizador/dispositivo receptor (por exemplo, smartphone, tablet, computador, etc.).

Requisitos não funcionais:

- O hardware deve ser compacto e passível de ser instalado nas infraestruturas actuais (com acesso à rede eléctrica) como, por exemplo, os mupis;

- O sistema deve funcionar com e sem acesso à Internet;

- A solução deve ter um custo acessível;

- A instalação da solução deve ser simples e prática.

Foi neste contexto que foi desenhada a proposta de arquitetura de sistema e que se carateriza pela existência de um ponto de acesso (figura 3) associado ao expositor do cartaz. Este ponto de acesso é um equipamento que disponibiliza uma rede sem fios que pode ser acedida por um conjunto variado de dispositivos receptores (nomeadamente móveis) necessários para consultar os conteúdos acessíveis, ou seja, a versão digital acessível do cartaz (figura 4).

Figura 3 Arquitetura de sistema: instalação do ponto de acesso

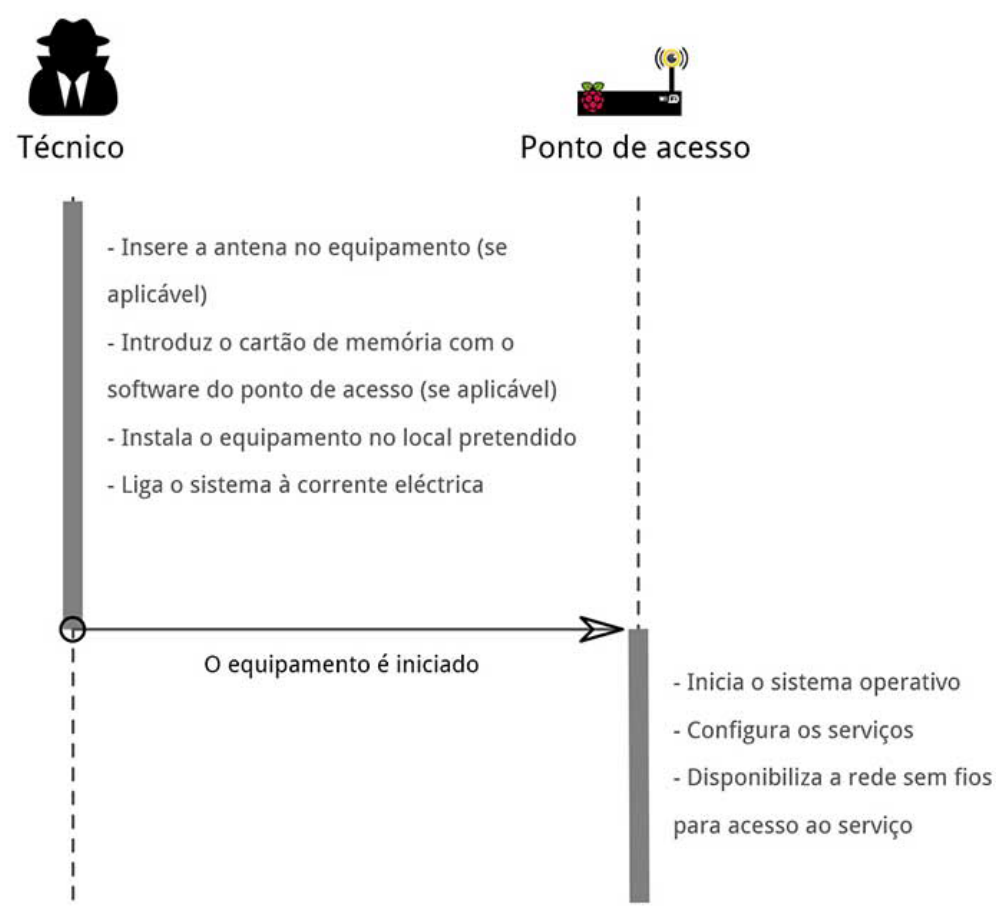


Figura 4 Ponto de acesso

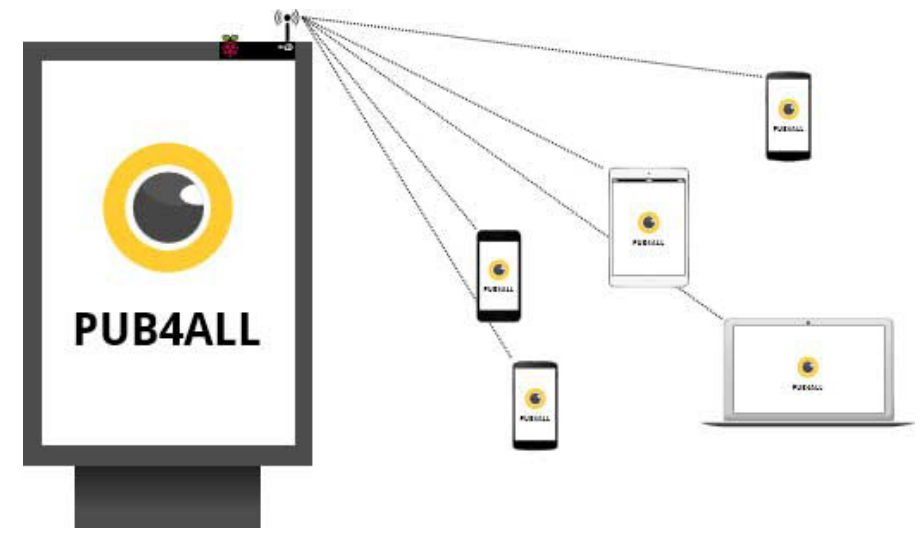

A versão digital do cartaz pode ser uma página ou aplicação móvel baseada em HTML (Hipertext Markup Language - Linguagem de Marcação de Hipertexto), uma norma aberta compatível com a maior parte dos dispositivos móveis de última geração que, para além dos recursos multimédia que associamos à Web, fornece também um conjunto de instruções semânticas específicas para ferramentas de acessibilidade como, por exemplo, leitores de ecrã. A transmissão de conteúdos, entre o cartaz e o dispositivo do recetor é baseada no protocolo HTTP (Protocolo de Transferência de Hipertexto) sob uma rede sem fios local disponibilizada pelo equipamento especificado.

Para a implementação do protótipo do ponto de acesso foram utilizados os seguintes componentes: um Raspberry Pi; uma caixa protectora para o Raspberry Pi; um cartão de memória de 16GB, um adaptador de redes sem fios USB e uma bateria portátil para alimentação da solução.

Neste cenário, os utilizadores utilizam os seus dispositivos cliente (telemóveis, por exemplo) para aceder à rede sem fios do ponto de acesso e, desta forma, conseguirem "ver" o cartaz. O acesso à informação do cartaz foi feita, no sistema prototipado (Imagem 5), através da leitura em áudio do ecrã: no momento em os dispositivos recetores cliente se aproximam do ponto de acesso, o dispositivo tenta associar-se à rede sem fios PUB4ALL e o utilizador recebe uma notificação com a informação da existência de um novo cartaz. $\mathrm{O}$ utilizador interage com a aplicação solicitando o cartaz digital, o ponto de acesso devolve uma página HTML e respectivos recursos sendo estes apresentados/lidos ao utilizador. 
Mineiro, J. P., Almeida, A. M. \& Lélis, C. | Cartazes urbanos acessíveis a pessoas cegas: proposta de um modelo de design para todos, baseado numa solução mobile

Figura 5 Modelo de sistema, apresentação do cartaz digital

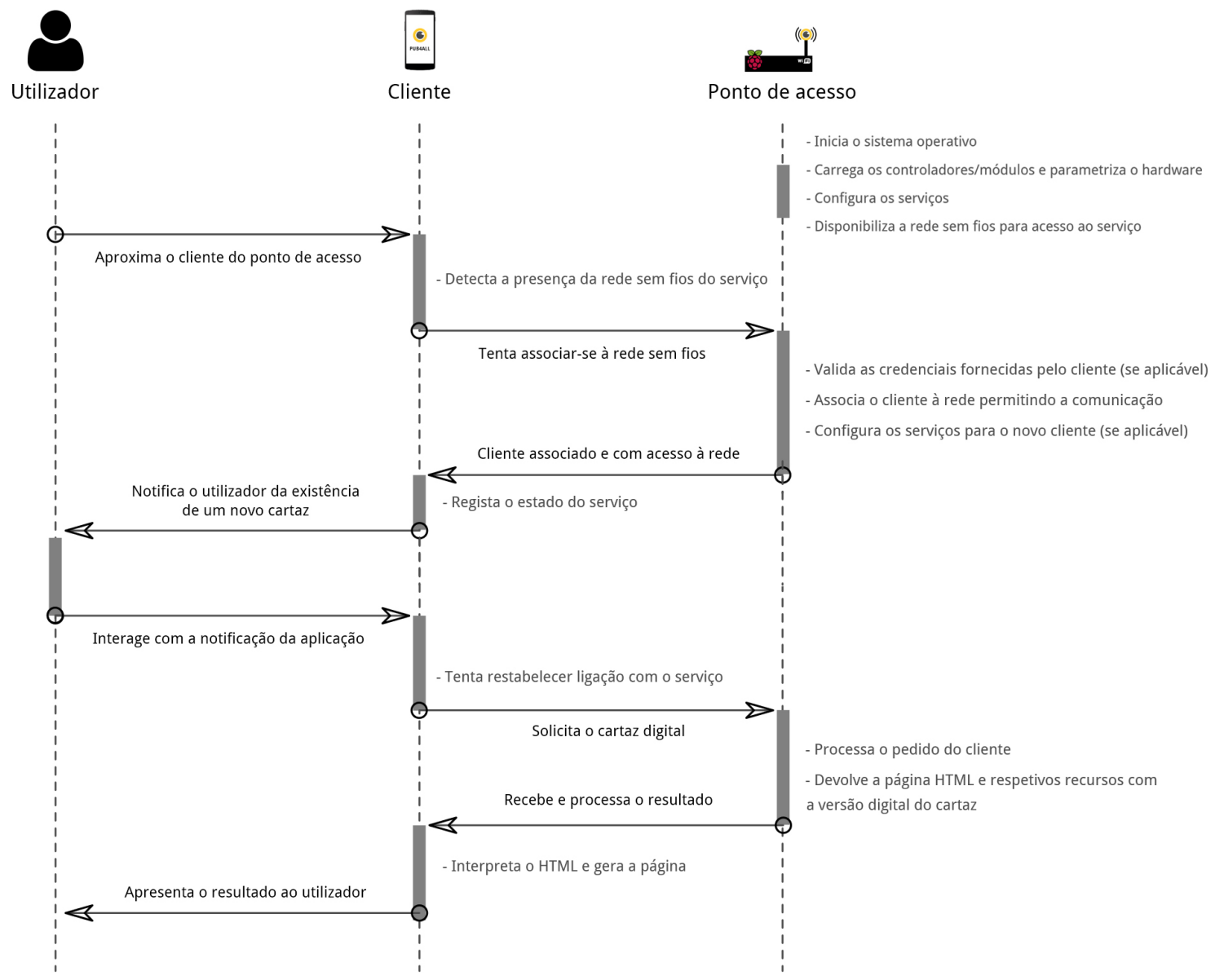

Caso a rede do serviços não esteja ao alcance do dispositivo, a aplicação mantêm-se em modo de espera até que uma nova lista de redes seja enviada pelo sistema. Esta funcionalidade, directamente associada aos mecanismos de gestão das redes sem fios do sistema operativo, permite economizar recursos e delegar no sistema operativo a responsabilidade desta gestão. 
Mineiro, J. P., Almeida, A. M. \& Lélis, C. | Cartazes urbanos acessíveis a pessoas cegas: proposta de um modelo de design para todos, baseado numa solução mobile

Figura 6 Interface da aplicação sem acesso a nenhum PUB4ALL e painel de opções da aplicação

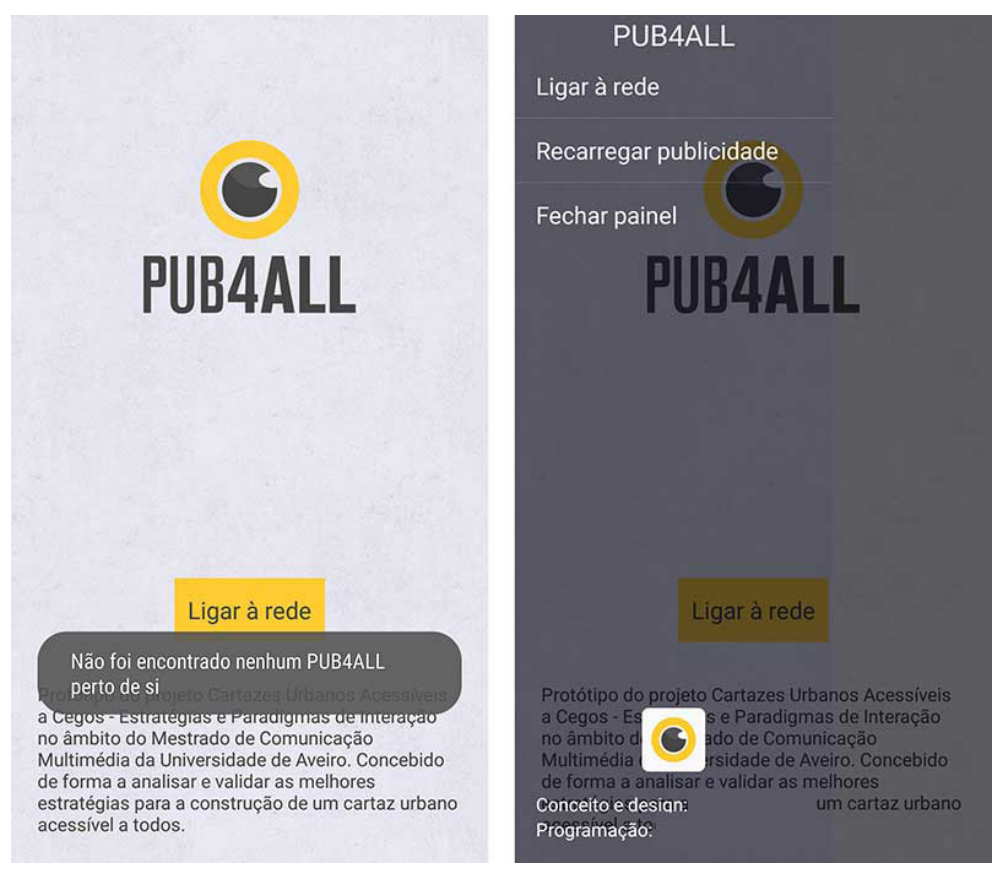

\section{Avaliação e análise}

Após a implementação do protótipo este foi submetido a uma série de testes para avaliar a viabilidade da solução proposta. Estes testes envolveram vários utilizadores multiplicados por vários dispositivos e sistemas, com e sem tecnologias assistivas, como por exemplo com ou sem leitor de ecrã.

Numa primeira fase foi instalada a aplicação móvel desenvolvida nos smartphones Android de alguns utilizadores e pediu-se que estes passassem junto do ponto de acesso para aferir se era emitida uma notificação pela aplicação; em resposta à notificação era apresentada versão digital do cartaz disponibilizada pelo ponto de acesso. Nos vários modelos testados (dois modelos da LG, um modelo da Samsung e dois modelos da Sony - num total de cinco equipamentos), o teste apenas falhou em dois equipamentos (um porque era uma versão de Android não suportada pela aplicação e outro devido a um problema com o equipamento). Assim, na maioria dos equipamentos testados, mesmo com diferentes resoluções e versões Android, o sistema funcionou tal como o previsto.

Para além dos testes com o cliente oficial para dispositivos Android, foram também efetuados alguns testes com outros equipamentos, nomeadamente dispositivos com o sistema operativo iOS, Windows, OSX e Linux. Neste cenário os equipamentos foram associados à rede sem fios aberta fornecida pelo serviço, tendo sido apresentada a janela de início de sessão em todos os sistemas 
operativos testados (com exceção do sistema operativo Linux que na distribuição utilizada não apresentou nenhuma interface para início de sessão na rede), com a apresentação do cartaz digital do serviço nesta janela. No caso do sistema operativo Linux, ao abrir um navegador Web e ao visitar um endereço o sistema reencaminha o utilizador para a versão digital do cartaz.

Figura 7 Cartaz físico com o protótipo do ponto de acesso para o cartaz digital

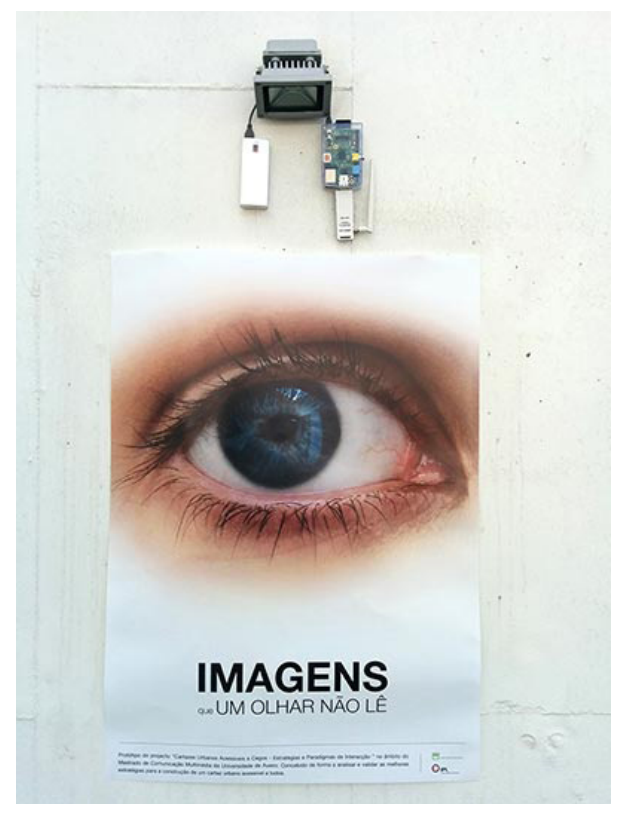

A última fase de testes foi feita com vários utilizadores com recurso ao mesmo equipamento (Imagem 7) e seguindo o mesmo percurso. Nesta fase tivemos um grupo de cinco pessoas cegas e duas pessoas normovisuais às quais foi pedido que colocassem no bolso (ou no local onde transportam habitualmente o seu equipamento móvel) um smartphone LG Nexus 5 com a aplicação Android desenvolvida no bolso ou no local onde transportam habitualmente o seu equipamento móvel e que seguissem o percurso já descrito; ao serem notificadas deveriam aceder ao dispositivo, interagir com a notificação e tentar interpretar a informação apresentada. Para as pessoas cegas foi ativado o leitor de ecrã do sistema (TalkBack) com uma voz fornecida pelo sistema no idioma Português do Brasil. Não foi dada qualquer indicação sobre o funcionamento da aplicação nem como utilizar o sistema.

Verificou-se que todos os utilizadores foram notificados da existência de um cartaz digital nas imediações do cartaz físico/ponto de acesso e que todos estes utilizadores conseguiram aceder à versão digital do cartaz e à informação nele contida, tanto no formato visual (para os normovisuais) como no formato áudio (para as pessoas cegas), e descrever essa informação.

Assim, com os testes realizados, verificou-se que a solução tem um grande potencial informativo, permitindo aceder a informação num formato mais acessível e inclusivo. 


\section{Conclusão e trabalhos futuros}

Pelos testes realizados é possível aferir que a solução proposta é funcional e tecnologicamente viável. Todos os utilizadores que participaram no teste reconheceram o valor e o potencial da mesma na promoção da sua inclusão digital e social. No entanto foi reconhecido pelos participantes neste estudo que, com recurso às potencialidades do sistema, esta solução pode ter um âmbito muito mais abrangente, adicionando valor não só para as pessoas com deficiência mas para todos os indivíduos.

Considerando a finalidade principal do trabalho, nomeadamente no que respeita ao estudo e identificação das melhores estratégias e paradigmas de interação a utilizar neste contexto, o trabalho desenvolvido permitiu comprovar a eficácia da utilização de dispositivos móveis associados a um modelo de notificação por aproximação física e leitura áudio dos cartazes.

Como trabalho a desenvolver no futuro pode ser equacionado um modelo de negócio que pressuponha um sistema para acesso à Internet para acesso exclusivo à página do promotor do cartaz e/ou acesso livre à Internet após ter sido consultada a publicidade/informação.

\section{Referências}

BERNS, ROBERTA (2002). O desenvolvimento da criança. São Paulo: Editora Loyola

CANZIANI, MARIA (1985). Educação Especial: Visão de um processo dinâmico e integrado. Fonte: Educa

DECLARAÇÃO DE ESTOCOLMO (2004). http://www.designforalleurope.org/ Design-for-All/EIDD-Documents/Stockholm-Declaration/ (20 de Março de 2015)

MOLES, ABRAHAM (2005). O cartaz. São Paulo: Editora Perspectiva S.A.

NORMAN, DONALD (2004). Emotional Design. Why we love (or hate) everyday things. New York: Basic Books

SHNEIDERMAN, BEN (2002). Leonardo's laptop: human needs and the new computing technologies. Massachusetts Institute of Technology

VAN DER MAREN, J. M. (1996) Méthodes de recherche pour léducation. ze édition, Bruxelles: De Boeck Université

VIANA, BRUNO (2010). Teasers- Imagens que provocam. Universidade fedral do Rio grande do sul, http://www.lume.ufrgs.br/handle/10183/27890 (11 de Fevereiro de 2015)

World Health Organization, 2014, WHO | Visual impairment and blindness, http://www.who.int/mediacentre/factsheets/fs282/en/ (26 de Fevereiro de 2015) 


\section{Sobre as autoras}

\section{Joana Pimparel Mineiro}

Joana Pimparel Mineiro natural de Leiria. Licenciada, em 2007, em Design Gráfico/Multimédia pela Escola Superior de Arte e Design das Caldas da Rainha do Instituto Politécnico de Leiria, terminou em 2015 Curso de Mestrado em Comunicação Multimédia - ramo Multimédia Interactivo na Universidade de Aveiro. Após a obtenção do grau de Licenciatura realizou um estágio no Jornal PÚBLICO, foi responsável pela paginação e design das revistas DaMoto, DaMotoClássica e DoPapel, no Grupo editorial "Agora Publicações e Comunicação" e desenvolveu trabalhos diversos na empresa Publicenso agência de Comunicação e Publicidade do Grupo Lena. Desde 2009 é responsável pelo Design Gráfico de conteúdos e plataformas na equipa da Unidade de Ensino a Distância do Instituto Politécnico de Leiria.

$<$ mineiro.joana@gmail.com>

\section{Ana Margarida Almeida}

$\mathrm{PhD}$ in Communication Sciences and Technologies and she has been developing research activities related to 'health promotion and health education through ICT', 'communication and health', 'digital inclusion', 'media for all' and 'agile projects development'. She is an Assistant Professor at the Department of Communication and Art, University of Aveiro, Portugal, she lectures in Communication Sciences and Technologies undergraduate, graduate and doctoral courses: 'New Communication Technologies bachelor degree', 'Multimedia Communications Master', 'Multimedia and Education PhD Program' and 'Information and Communication in Digital Platforms PhD Program". Under this scope, she has developed several supervising activities of MSc and PhD students, highly related to her main research interests and she has been involved in different research projects and scientific initiatives. She has several papers and book chapters published and she is also a reviewer in several national and international journals.

<marga@ua.pt> 
Mineiro, J. P., Almeida, A. M. \& Lélis, C. | Cartazes urbanos acessíveis a pessoas cegas: proposta de um modelo de design para todos, baseado numa solução mobile

\section{Catarina Lélis}

Catarina Lélis is an invited assistant professor at the Department of Communication and Art in the University of Aveiro, Portugal. She holds a Masters of Innovation and Knowledge Management and previously she studied Communication Design. She has had several years of Brand Design and Identity Consultancy experience in a range of industries. She is the author of several technical manuals to support the learning of graphic and design applications (such as Freehand, Illustrator, InDesign). Her research and teaching interests lie within Participatory Design, Brand Management and Corporate Identity within New Media and the Social Web.

$<$ lelis@ua.pt $>$

Artigo recebido em 21 mar. 2015, aprovado em 18 jun. 2015. 
Mineiro, J. P., Almeida, A. M. \& Lélis, C. | Cartazes urbanos acessíveis a pessoas cegas: proposta de um modelo de design para todos, baseado numa solução mobile 